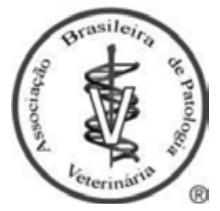

\title{
Anatomopathological, ultrastructural, immunohistochemical and molecular characterization of infectious laryngotracheitis outbreaks in poultry farms in Egypt (2018-2020)
}

\author{
Mohamed El-Saied ${ }^{1}$, Magdy El-Mahdy ${ }^{1}$, Ezz El-Din Sakr ${ }^{1}$, Mostafa Bastami $^{2}$ \& Mohamed Shaalan ${ }^{1 *}$ \\ ${ }^{1}$ Department of Pathology, Faculty of veterinary medicine, Cairo University, 12211 Giza, Egypt \\ ${ }^{2}$ Department of Poultry Diseases, Faculty of veterinary medicine, Cairo University, 12211 Giza, Egypt \\ *Corresponding author: Dr. Mohamed Shaalan. Department of Pathology, Faculty of veterinary medicine, \\ Cairo University, 12211 Giza, Egypt. E-mail: mohamedibrahim@cu.edu.eg
}

Submitted February, $1^{\text {st }}$ 2021, Accepted April, 22 2021

\begin{abstract}
Infectious laryngotracheitis (ILT) is a severe respiratory disease, which causes high morbidity and mortality in affected birds. In our study, ILT were reported in 42 farms from nine governates over two years (2018-2020) that showed clinical signs of ILT including dyspnea, blood expectoration, excessive lacrimation, rattling, conjunctivitis. The disease affected different chicken breeds and age groups despite vaccination with licensed and commonly used vaccines. Samples of larynx, trachea, lungs and air sacs were examined and collected for histopathological, ultrastructural, immunohistochemical examination and molecular detection. Gross examination of laryngeal and tracheal lumen revealed different types of exudates varied from catarrhal to fibrinonecotric, also pneumonia and airsacculitis were detected. Histopathological examination showed different alternation in larynx, trachea, lung and air sac as characteristic syncytial cells containing intranuclear inclusion body hanged in fibrinoheterphilic exudate that precent in laryngeal, tracheal, bronchial and parabronchial lumen and air sacs. Tracheal lesion scoring system was used to categorize the severity of lesion in different governates. Tracheal lesion score showed that $6.02 \%, 26.5 \%, 43.3 \%$ of the birds exhibited mild, moderate, and severe changes, respectively, while $24.18 \%$ of the birds exhibited very severe changes. Furthermore, severe cases were related to the Qalyubia , Fayoum then Sharkia Governorate. Moreover, immunohistochemistry was used to detect viral particles in syncytial cells, inflammatory cells beside epithelium of trachea and lung. Transmission electron microscopy enabled the detection of virus particles and demonstrated that heterophils could be infected. PCR targeting a region in the thymidine kinase gene and glycoprotein gJ gene confirmed the presence of infectious laryngotracheitis ILT virus-specific DNA. In conclusion, anatomopathological, immunohistochemical, molecular and ultrastructural findings showed increased of ILTV severity in Egypt. Larynx, trachea, lungs and air sac should be collected and examined that aid in diagnosis. Importance of good biosecurity level to be considered.
\end{abstract}

Key words: ILTV, histopathology, immunohistochemistry, electron microscopy, PCR, Egypt.

Introduction

Infectious laryngotracheitis (ILT) is a severe acute respiratory viral disease affecting chickens and causing respiratory manifestations such as dyspnea, bloody expectoration, swelling of paranasal sinuses, and conjunctivitis. ILT is characterized by high morbidity and mortality with decreased egg production in laying hens, resulting in a substantial negative impact on the poultry industry (13).
Infectious laryngotracheitis virus (ILTV), also known as Gallid herpesvirus-1 (GaHV-1), is a member of Alphaherpesvirinae family (27) and has a worldwide distribution. The morbidity and mortality rates differ according to the virulence of the circulating strains $(17,20,21)$.

Gross lesions of ILT can be observed in the larynx and trachea as mucoid or bloody casts obstructing the laryngeal and tracheal lumina, conjunctivitis, swelling of the infraorbital sinuses, and nasal discharge (22). Other lesions are observed in the lungs including congestion and airsacculitis (34). 
Histopathology is a key diagnostic tool in the ILT detection, specifically in the acute phase of the infection. As a result of ILT infection, thickening of the laryngeal and tracheal mucosa with vascular changes, inflammatory edema and mononuclear cells infiltrate may typically be observed. A diagnostic hallmark is the formation of multinucleated cells (syncytia) with intranuclear inclusion bodies present in both laryngeal and tracheal epithelial cells or in fibrinoheterophilic cast. With disease progression, necrosis and desquamation of the laryngeal and tracheal epithelium may be observed $(6,23)$

The control of ILT can be approached by vaccination of birds either by using chicken embryo origin vaccine (CEO), by tissue-culture origin (TCO) vaccine or by the recombinant subunit vaccine based on fowl pox virus (FPV) as vectors to express ILTV immunogens for protection against ILT (14). Bird-to-bird passage, excessive vaccine uses and passage in non-vaccinated birds can revert the virulence of the vaccine strains which could increase the virulence of the ILT virus (ILTV) and cause outbreaks (12, 22) or disease reactivation (16).

The virus may persist in the trigeminal ganglia for 15 months after vaccination and can be detected in vaccinated birds two days after vaccination. $(26,33)$. Shedding of the virus increases when birds are exposed to different stress factors or when birds are mixed with unknown reservoirs birds, which may result in latent infection in predisposed birds $(16,33)$.

The differentiation between wild-type and vaccinal ILTV strains is difficult because of the antigenic and genetic homogeneity (11). Several studies have documented vaccinal outbreaks occurring all over the world. Liveattenuated virus vaccines can prompt a variety of clinical signs of disease, including tracheal lesions, and mortality that varies according to the strain of the virus used in the vaccine and the age of affected birds (21).

In Egypt, at late 1982 and early 1983, ILTV was first recorded by Tantawi et al. (31), and protection of chickens against ILTV was launched using CEO and TCO vaccines. The vaccine is applied via different routes such as drinking water or eye drops using either single dose when the chickens are approximately 35-45 days of age, or twice when the chickens are approximately $35-55$ days and $95-115$ days of age $(13,29)$. Furthermore, in some farms, TCO and CEO vaccines have been used in the same flock during the breeding period (18).

Shehata et al. (29) found that outbreaks of ILTV were related to circulating CEO-like strains. Ali et al. (2) suggested that CEO-ILT vaccine viruses could be the source of the outbreak occurring in commercial layer flocks in Sharkia, with the strain becoming more virulent following bird-to-bird passages, resulting in severe outbreaks in susceptible birds in Egypt. Bayoumi et al. (4) reported that both CEO-vaccinelike and recombinant virus strains were circulating in 2018 and 2019 and related to outbreaks in Egypt.

A study in the USA found that $75 \%$ of the ILTV strains isolated from field outbreaks were related to CEO vaccine strains (8). New virulent ILTV strains were reported as a recombination between attenuated vaccine strains in the field $(1,19)$. In Australia, whole genome analysis of an ILTV isolate revealed that recombination is a continuous process, leading to the emergence of virulent strains. The isolate was suggested to be a recombinant of vaccine strain and another recombinant virus (28).

New outbreaks of ILT are emerging in many parts of the world due to extensive use of live attenuated vaccines. Previous experimental studies suggested exacerbated prolonged ILT infections following poor mass CEO vaccination (9).

The aim of this study was to collect samples from nine Egyptian governorates in order to:

a. Describe gross and microscopic respiratory tissues affinity of chickens infected with ILTV.

b. Apply different methods of virus detection, including immunohistochemistry, transmission electron microscopy and PCR

c. Compare ILT severity in different locations in Egypt by tracheal histopathological lesion scoring.

\section{Materials and methods}

\section{Sampling}

Samples were taken from farms showed clinical signs of ILT. A total of 166 tissue samples from freshly dead and live birds were collected from 42 poultry farms of sasso, layer and breeder chickens located across nine governorates in Egypt. The age of sasso ranged from 40 to 75 days, layers from 160 to 250 days and breeders broilers from 180 to 300 days. All birds were vaccinated against Newcastle disease, infectious bursal disease, Marek's disease, avian influenza and infectious bronchitis virus. Moreover, twenty-five layer farms and five breeder broiler farms were vaccinated with both chicken-embryo origin (CEO) and tissue-culture origin (TCO) vaccines, while twelve sasso farms were vaccinated with $\mathrm{CEO}$ vaccine.

The birds were submitted to our clinic for examination during the period of 2018 to 2020. Each case was subjected to routine clinical and postmortem examination. The flock history, clinical signs, and gross lesions were recorded in addition to histopathological findings. Clinical signs were observed in all living birds $(n=120)$ included conjunctivitis with respiratory signs varied from mild to severe dyspnea and hemorrhaging from the oropharyngeal cavity and nostrils.

The animal handling and sampling procedures were performed according to the guidelines regulated by the Institutional Animal Care and Use Committee in the Faculty of Veterinary Medicine, Cairo University (VetCU01102020217), Egypt. Samples of larynx, trachea, lung, and air sac samples were fixed in 10\% neutral-buffered formalin, and aseptically collected in the field and transported under refrigerated conditions for molecular studies. (Table 1). 
Table 1. Number of sampled flocks and birds in nine different governorates in Egypt

\begin{tabular}{ccc}
\hline Governorate & $\begin{array}{c}\text { No. of } \\
\text { sampled flocks }\end{array}$ & $\begin{array}{c}\text { No. of } \\
\text { sampled Birds }\end{array}$ \\
\hline Qalyubia & 20 & 75 \\
Fayoum & 5 & 30 \\
Mansoura & 5 & 20 \\
Sharqia & 5 & 20 \\
Alexandria & 3 & 10 \\
Giza & 1 & 2 \\
Gharbia & 1 & 3 \\
Damietta & 1 & 2 \\
Ismailia & 1 & 4 \\
\hline
\end{tabular}

\section{Histopathological examination}

Larynx, trachea, lung, and air sac samples were kept in 10\% neutral-buffered formalin for $24 \mathrm{~h}$. Then, the samples were washed, dehydrated in ascending grades of alcohol, and embedded in paraffin blocks, and 4- $\mu \mathrm{m}$ tissue sections were prepared and stained with hematoxylin and eosin (H\&E) for routine histopathological examination (3). Lesion scores of tracheal samples were calculated according to Koski et al. (18) with modification (Table 2).

\section{Immunohistochemical analysis}

Twenty samples of lung and trachea from different farms that showed severe clinical signs and histopathological lesion score were applied for immunohistochemistry. All the immunohistochemical procedures were performed as previously described by Yavuz et al. (34). Tissue sections of lung and trachea were incubated overnight with rabbit polyclonal anti-ILTV-gE antibody diluted 1:400 (Cat.no. orb10560, Biorbyt company, Cowley Road, Cambridge, United Kingdom), washed and incubated for one hour with SM802 EnVision ${ }^{\mathrm{TM}}$ Flex /HRP 3 x $40 \mathrm{~mL}$ (Dako, Santa Clara, USA), ready-to-use dextran coupled with peroxidase molecules and goat secondary antibody. Next, the sections were washed, and immune reactivity was detected by DM827 EnVision ${ }^{\mathrm{TM}}$ Flex DAB + Chromogen (Dako) DAB staining and counterstaining with Mayer's hematoxylin. Brown staining observed by light microscopy indicated immune reactivity. Samples from SPF chickens was considered as a negative control.

\section{Detection of ILTV by transmission electron microscopy}

Five tracheal samples were cut into cubes of $1 \mathrm{~mm} 3$ and were fixed in 3\% cold glutaraldehyde then washed 3 - 4 times by $0.1 \mathrm{M}$ sodium cacodylate buffer, $\mathrm{pH} 7.4$ for 20 minutes every time and were re-fixed in $1 \%$ osmium tetraoxide in $0.1 \mathrm{M}$ sodium phosphate buffer for two hours. Next, dehydrated by their immersion in ascending grades of ethanol (50-90\%) for 15 minutes each was followed with absolute alcohol for 15 minutes and then embedded in the epon- araldite mixture. From the embedded blocks semithin sections by LKB ultramicrotome in thickness of $0.5-1$ micron were prepared for orientation of the tissue and photographed by sc30 Olympus camera and then ultrathin section in thickness of 50-70 nm were made using Leica AG ultramicrotome and contrasted in uranyl acetate and lead citrate, examined by JEOL JEM 100 CXII (JEOL Ltd, Tokyo, Japan) electron microscope at $80 \mathrm{KV}$ and photographed by CCD digital camera Model XR- 41 as described previously by Crespo et al.(6)

\section{Molecular detection of ILTV}

Total DNA was extracted from tracheal samples of five randomly sampled farms. The samples of each farm were pooled together and extracted using the QIAamp DNA Mini Kit (Qiagen, Hilden, Germany) according to the manufacturer's protocol to confirm ILT diagnosis. The thymidine kinase (TK) gene and gJ gene of ILTV were targeted for the detection of ILTV by PCR. EmeraldAmp GT PCR Master Mix (Takara, Kusatsu, Japan) used in the reaction that contained 10 pmol of each primer, $1 \mathrm{U}$ of TaKaRa Ex Taq (Takara Bio Inc., Kusatsu, Japan), and $200 \propto \mathrm{M}$ of each deoxynucleotide (Takara Bio Inc.).

Table 2. Tracheal lesion score system used in the study.

\begin{tabular}{ll}
\hline Lesion score & Histopathological changes \\
\hline Score 1 & $\begin{array}{l}\text { mild changes characterized by increased mucosal thickness with moderate cell infiltration and some sloughed } \\
\text { epithelium. Hyperemia is present. }\end{array}$ \\
\hline Score 2 & $\begin{array}{l}\text { moderate changes as mucosa thickened by marked cell infiltration and edema. Patches of affected epithelium } \\
\text { sloughed from lamina propria with syncytial cells containing intranuclear inclusion bodies. Marked } \\
\text { hyperemia, cuffs of mono-nuclear cells around vessels outside mucosa }\end{array}$ \\
\hline Score 3 & $\begin{array}{l}\text { severe changes in which mucosa thickened because of edema, proteinaceous fluid, cellular exudate, or } \\
\text { adherent fibrinohemorrhagic to cellular pseudomembrane on the surface, syncytia with inclusion bodies are } \\
\text { also present }\end{array}$ \\
\hline Score 4 & Very severe changes mucosa has no residual epithelium and mononuclear cells infiltrations \\
\hline
\end{tabular}


Table 3. Primers sequences used in the study.

\begin{tabular}{|c|c|c|c|c|}
\hline Primers & Sequence (5'- 3') & Amplification conditions & Amplicon size (kb) & Reference \\
\hline TK-F & CTGGGCTAAATCATCCAAGACATCA & \multirow{2}{*}{$\begin{array}{c}94^{\circ} \mathrm{C}(5 \mathrm{~min}), 40 \times\left(94^{\circ} \mathrm{C} 60 \mathrm{sec} ; 60^{\circ} \mathrm{C} 30\right. \\
\left.\sec ; 72^{\circ} \mathrm{C} 120 \mathrm{sec}\right), 72^{\circ} \mathrm{C} 10 \mathrm{~min}\end{array}$} & \multirow{2}{*}{2.24} & \multirow{2}{*}{-17} \\
\hline TK-R & GCTCTCTCGAGTAAGAATGAGTACA & & & \\
\hline gJ-F & ATTTCGCCGAGAGATGGGGAC & \multirow{2}{*}{$\begin{array}{c}94^{\circ} \mathrm{C}(5 \mathrm{~min}), 40 \times\left(94^{\circ} \mathrm{C} 30 \mathrm{sec} ; 53^{\circ} \mathrm{C} 30\right. \\
\left.\text { sec; } 72^{\circ} \mathrm{C} 90 \mathrm{sec}\right), 72^{\circ} \mathrm{C} 10 \mathrm{~min}\end{array}$} & \multirow{2}{*}{1.39} & \multirow{2}{*}{-5} \\
\hline gJ-R & CAGTGTATTTTCTGACTCACCG & & & \\
\hline
\end{tabular}

Table 3 described the cycling program for each amplification that obtained in a Gene-Amp 9700 thermal cycler (Applied Biosystems, Foster City, CA). Positive control obtained from DNA of attenuated ILTV live vaccine (LT-IVAX strain). SPF chicken tissues was used as a negative control. Electrophoresis in a $0.8 \%$ agarose gel stained with ethidium bromide were used for separation of specific PCR product that visualized by a Gel Doc XR gel documentation system (Bio-Rad Laboratories, Milan, Italy).

\section{Results}

\section{Clinical signs and gross pathology}

The disease affected different chicken breeds and age groups despite vaccination with commonly used vaccines .These farms showed showed poor biosecurity level with high stocking density and bad ventilation .
The examined birds exhibited respiratory signs manifestations, swelling of the paranasal sinuses, severe decrease in egg production and high mortality rates. The observed clinical signs of the affected birds were directly related to the severity of the viral infection.

Gross lesions were observed in larynx and trachea categorized into mild tracheitis that characterized by hyperemic mucosa with catarrhal exudate (Fig. 1A), moderated tracheitis showed hyperemic mucosa with bloody exudate (Fig. 1B) and severe tracheitis fibrinonecrotic exudate adhered to mucosa were detected (Fig. 1C). Caseous plug obstructing laryngeal and tracheal lumen were seen as in (Fig. 1D). In some cases, bronchi were occluded by mucous or and caseous plugs. Pulmonary congestion was observed with consolidated areas in some cases. Air sacs were turbid and thickened were noted in some cases. Different gross lesion listed in the following table (Table 4) with the number of cases out of total number of examined birds.
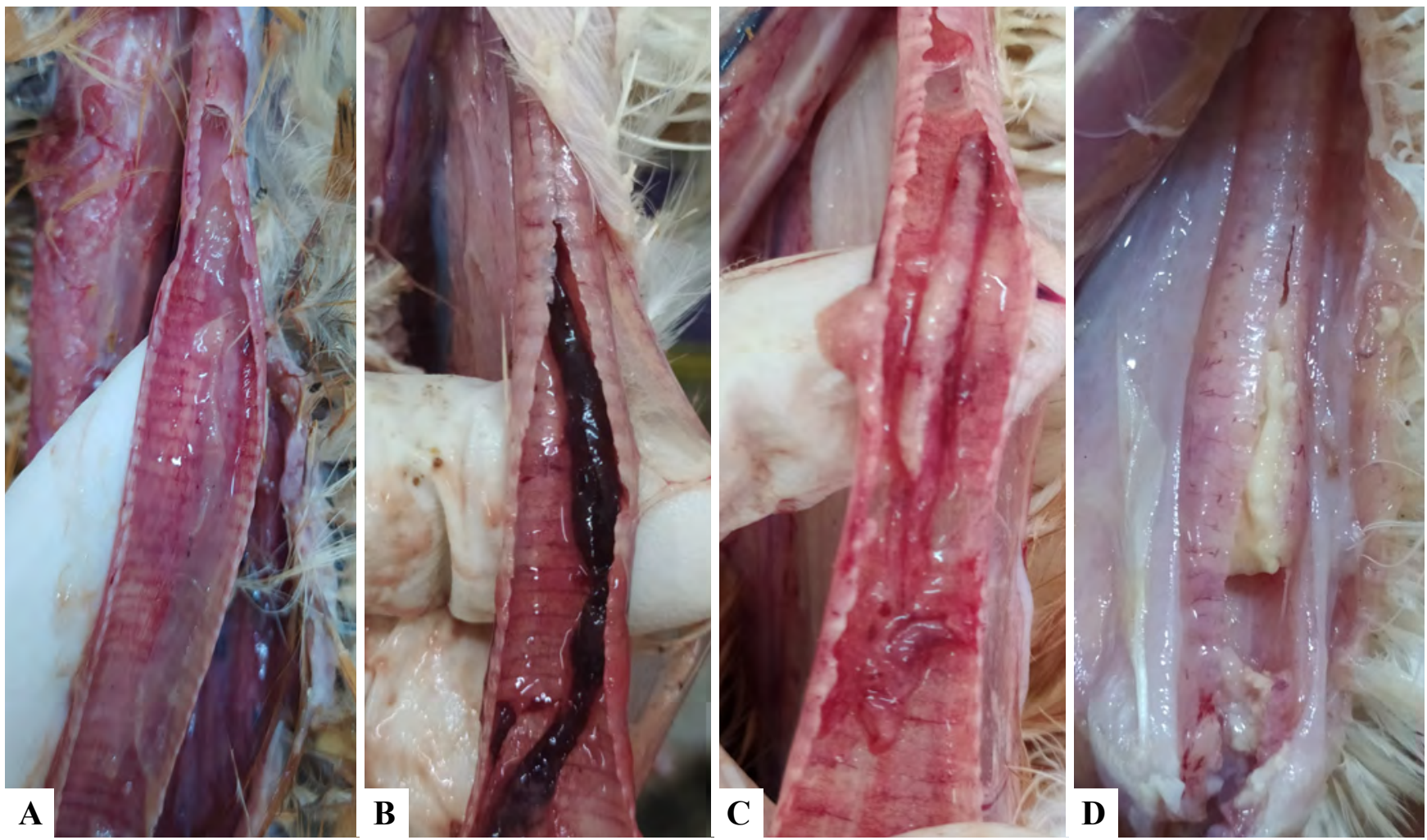

Figure 1. Gross lesions of trachea in ILTV-infected birds. A. Presence of catarrhal exudate in hyperemic tracheal lumen. B. The tracheal lumen is filled with bloody cast. C.Fibrinonecrotic exudate (pseudomembrane) adhere to hyperemic tracheal mucosa. D. The laryngeal and tracheal lumen is occluded with caseous material. 
Table 4. Recorded gross lesion with the number of cases with observed lesion

\begin{tabular}{ccc}
\hline Gross pathology & No. of cases/total cases & Percent \\
\hline Catarrhal tracheitis & $60 / 166$ & 36.1 \\
Hemorrhagic tracheitis & $82 / 166$ & 49.3 \\
Fibrinonecrotic tracheitis & $65 / 166$ & 39.1 \\
Occluded laryngeal and tracheal lumen (caseated) & $80 / 166$ & 48.1 \\
Occluded bronchi lumen & $60 / 166$ & 36.1 \\
Pulmonary congestion & $75 / 166$ & 45.1 \\
Airsacculitis & $65 / 166$ & 39.1 \\
\hline
\end{tabular}
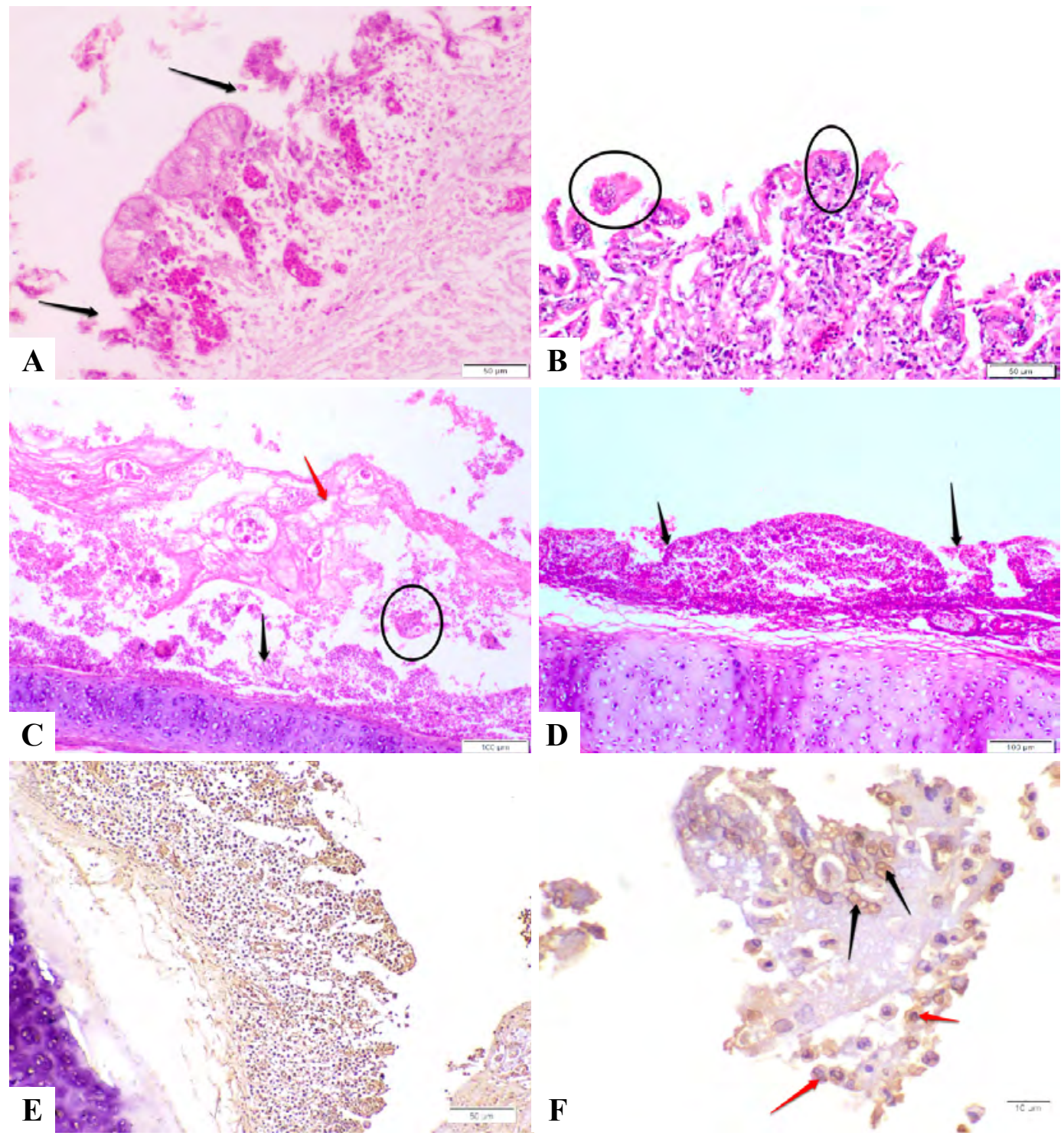

Figure 2. Tracheal microscopical lesion scores and immunohistochemistry in ILTV infected chickens. A. Score 1 showing sloughing of tracheal epithelium (arrow) and marked hyperemia with inflammatory cells infiltration tracheal mucosa. B. Score 2 syncytial cells with intranuclear inclusion bodies (circle) are detected in tracheal lining epithelium with hyperemia. C. Score 3 tracheal lumen is filled with marked fibrinoheterohilic exudate (red arrow) containing numerous syncytial cells (Circle) also denuded tracheal epithelium is noticed (black arrow). D. Score 4 complete ulcerated tracheal mucosa (arrow) with hemorrhage and mononuclear cells infiltration is present. E. Positive immunoreactivity is detected in the epithelial lining of the trachea as evidenced by yellowish brown granules alongside with inflammatory cells infiltration in the lamina propria (IHC). F. Intranuclear positive immunoreactivity present in syncytial cells (black arrow) and also in inflammatory cells in tracheal lumen (red arrow) (IHC). 


\section{Histopathological examination}

Histopathological examination of ILT infected birds showed thickening of the mucosa and submucosa of larynx and trachea by vascular changes such as congestion and inflammatory exudate as well as dense mononuclear cell infiltrates was observed at laryngeal and tracheal mucosa and submucosa (Fig. 2A). Detached epithelium in the tracheal lumen formed syncytial cells contained several intranuclear and eosinophilic inclusion bodies were detected (Fig. 2B). In addition, epithelial necrosis, hyperplasia, and desquamation, along with fibrin, free erythrocytes, cellular debris and few heterophils formed a fibrinonecrotic exudate filing the tracheal lumens (Fig. 2C). A sloughed and denuded tracheal epithelium was detected in very severe cases (Fig. 2D).
The lungs of affected birds showed (focal, multifocal, or diffuse) lymphoid hyperplasia and vascular changes including congestion with perivascular edema as well as marked interstitial expansion with edema, fibrin, heterophilic and lymphohistiocytic exudates caused disruption of the lung parenchyma.

Moreover, we observed acute fibrinous bronchopneumonia characterized by diffuse fibrinous exudate admixed with heterophils, desquamated epithelium and syncytial cells containing numerous intranuclear and eosinophilic inclusion bodies in the primary bronchi and parabronchial lumina (Fig. 3A and 3B) extending to pleura (Fig. 3C). Air sacs were thickened including hyperplastic epithelium, fibrin, inflammatory mononuclear cells and syncytial cells.

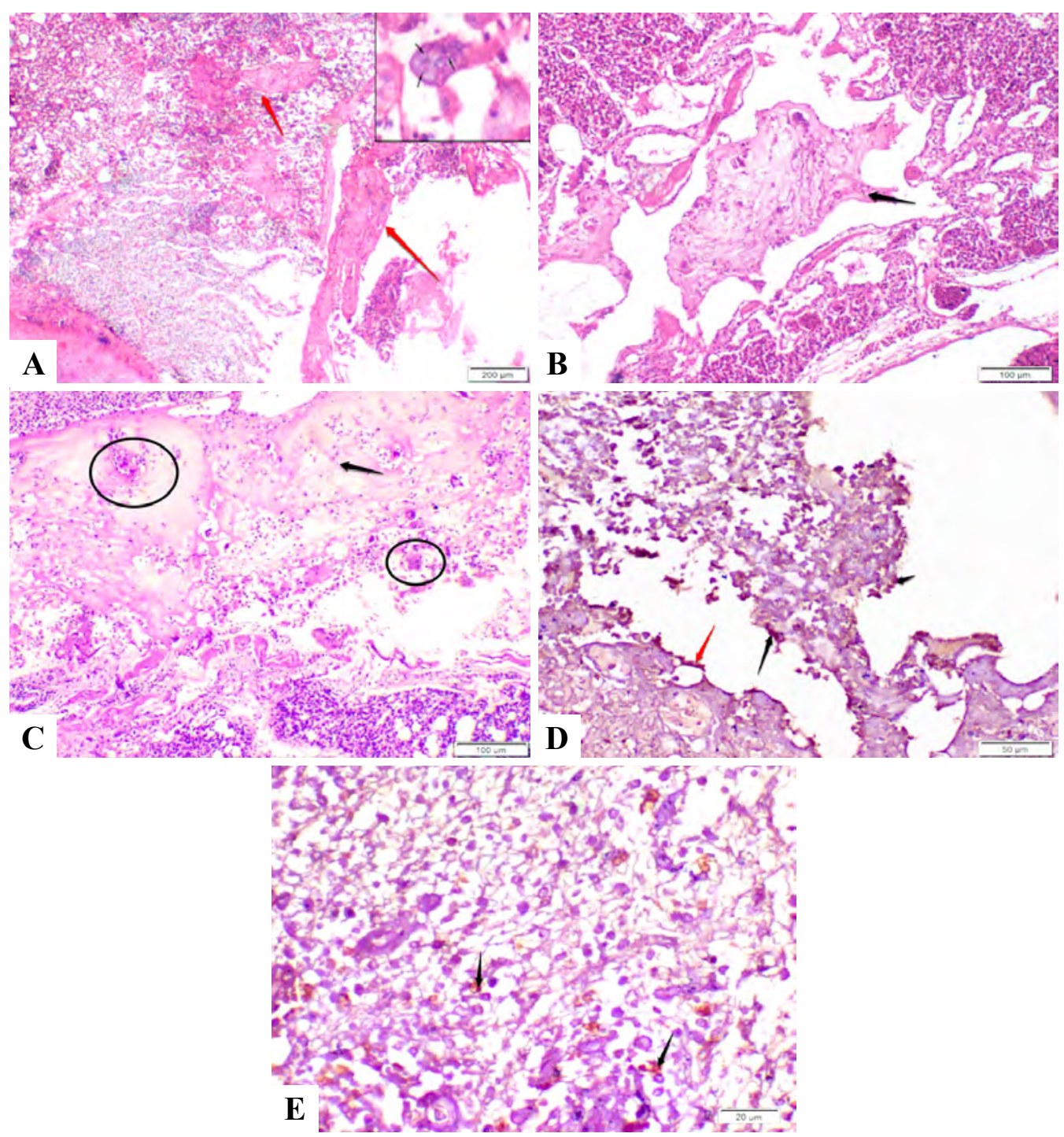

Figure 3. Histopathology and immunohistochemistry of lung and pleura in ILTV-infected birds. A. Primary bronchus is filled with fibrinoheterophilic exudate (red arrows) mixed with inflammatory cells, erythrocyte, denuded epithelium with numerus syncytial cells containing intranuclear inclusion bodies (insert). B. Fibrioheterophilic exudate (arrow) occult parabronchus lumen C. Thickening of the pleura and fibrinoheterophilic exudate (arrow) with several epithelial syncytial cells(circle). D. Immunoreactivity in the epithelial lining of the parabronchus (red arrow) and intraluminal inflammatory cells (black arrow) E. Inflammatory cells infiltrating the pleura and exhibiting positive immunoreactivity (arrows). 
Table 5. Histopathological lesion detected in different organs.

\begin{tabular}{|c|c|c|}
\hline Histopathological lesion & No. of cases/total cases & Percent \\
\hline Tracheal Inflammation & $149 / 166$ & 89.7 \\
\hline Tracheal necrosis & $129 / 166$ & 77.7 \\
\hline Tracheal Inclusions & $125 / 166$ & 75.3 \\
\hline Chronic tracheitis (Regenerative) & $20 / 166$ & 12 \\
\hline Pulmonary congestion and edema & $100 / 166$ & 60.2 \\
\hline Pulmonary Inclusion & $60 / 166$ & 36.1 \\
\hline Airsacculitis & $65 / 166$ & 39.1 \\
\hline Airsac Inclusions & $7 / 166$ & 4.2 \\
\hline
\end{tabular}

Tracheal lesions were the most characteristic lesions of ILTV-infected birds. The Lesions were scored and summarized Table 5 according to degree of severity and histopathological findings.

Overall, only $6.02 \%$ of the birds with a score 1 that exhibited mild changes (10 cases); $26.5 \%$ of the birds showed moderate changes with a score 2 (44 cases), $43.3 \%$ of the birds showed severe changes that scored 3 ( 72 cases), and $24.18 \%$ of the birds exhibited very severe changes that scored 4 (40 cases) (Fig. 4).

\section{Immunohistochemical analysis}

Immunoreactivity was noted in tissue sections from the larynx, trachea, lungs, pleura, and air sacs. Viral particles were detected in both laryngeal and tracheal epithelial cells, in the infiltrating inflammatory cells in lamina propria. Intranuclear positive reactivity was observed in syncytial cells with intracytoplasmic reactivity in inflammatory cells that present in laryngeal and tracheal lumen (Fig. 2E and 2F). In the lung, intracytoplasmic immunoreactivity was detected in the lining epithelium of the bronchus and parabronchus, in addition to inflammatory cells in their lumen and in the pleura (Fig. 3D and 3E). No immunoreactivity was detected in the larynx, trachea and lung of SPF chicken.

\section{Detection of ILTV by transmission electron microscopy}

The TEM examination and imaging of the tracheal epithelial mucosa showed the presence of electron-dense viral particles surrounded by a hollow zone, which is suggestive of ILT virus in both nucleus and cytoplasm (Fig. 5A and 5B). Moreover, heterophils in blood vessels in the tracheal mucosa showed the presence of electron-dense viral particles surrounded by a hollow zone in its cytoplasm (Fig. 5C and 5D).

\section{Molecular detection of ILTV}

Samples collected from birds showing clinical signs and histopathology suggestive for ILT were pooled and investigated for detection of ILTV by PCR targeting a 2.24-kbp fragment of the TK gene and a 1.39-kbp portion of the $\mathrm{gJ}$ gene. All five samples were positive for both genes (Fig. 6A and 6B).

Tracheal lesion score

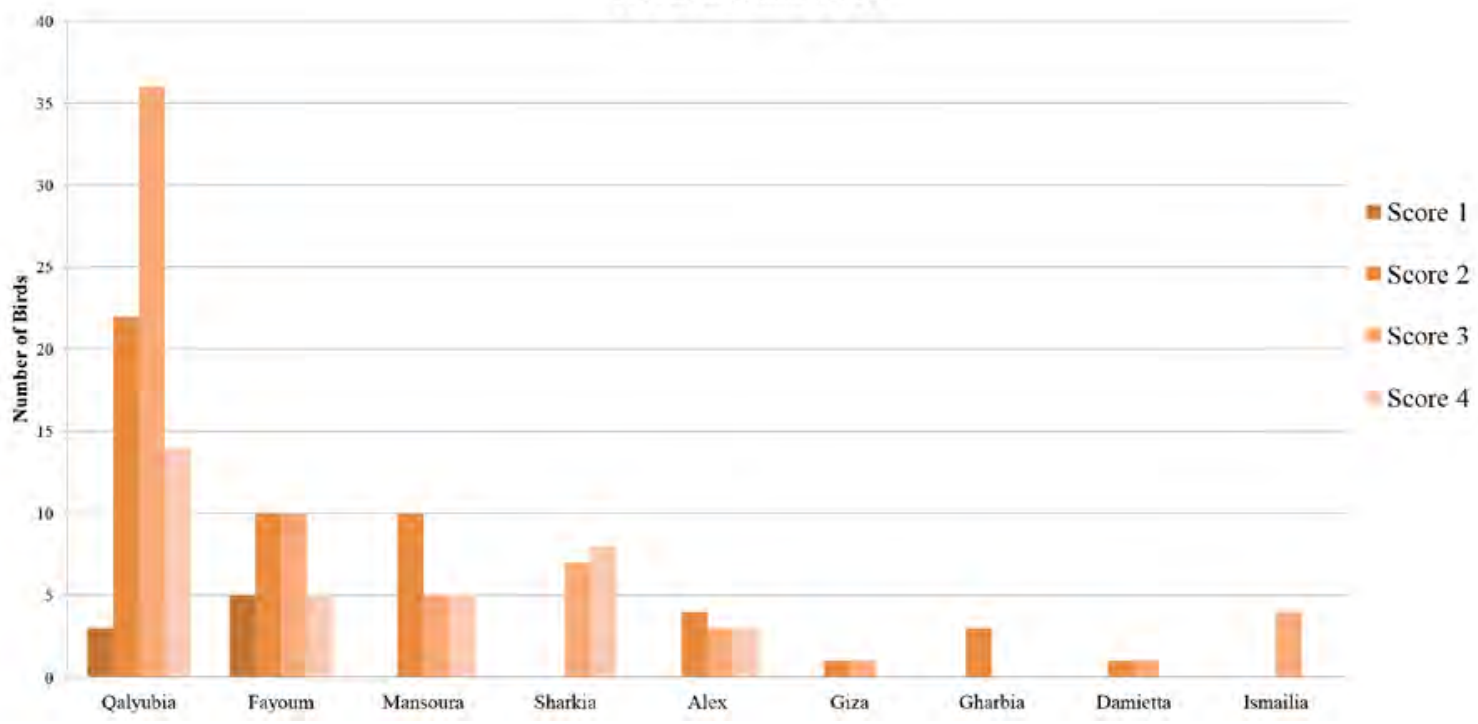

Figure 4. Chart showing the tracheal lesion score in different governorates 

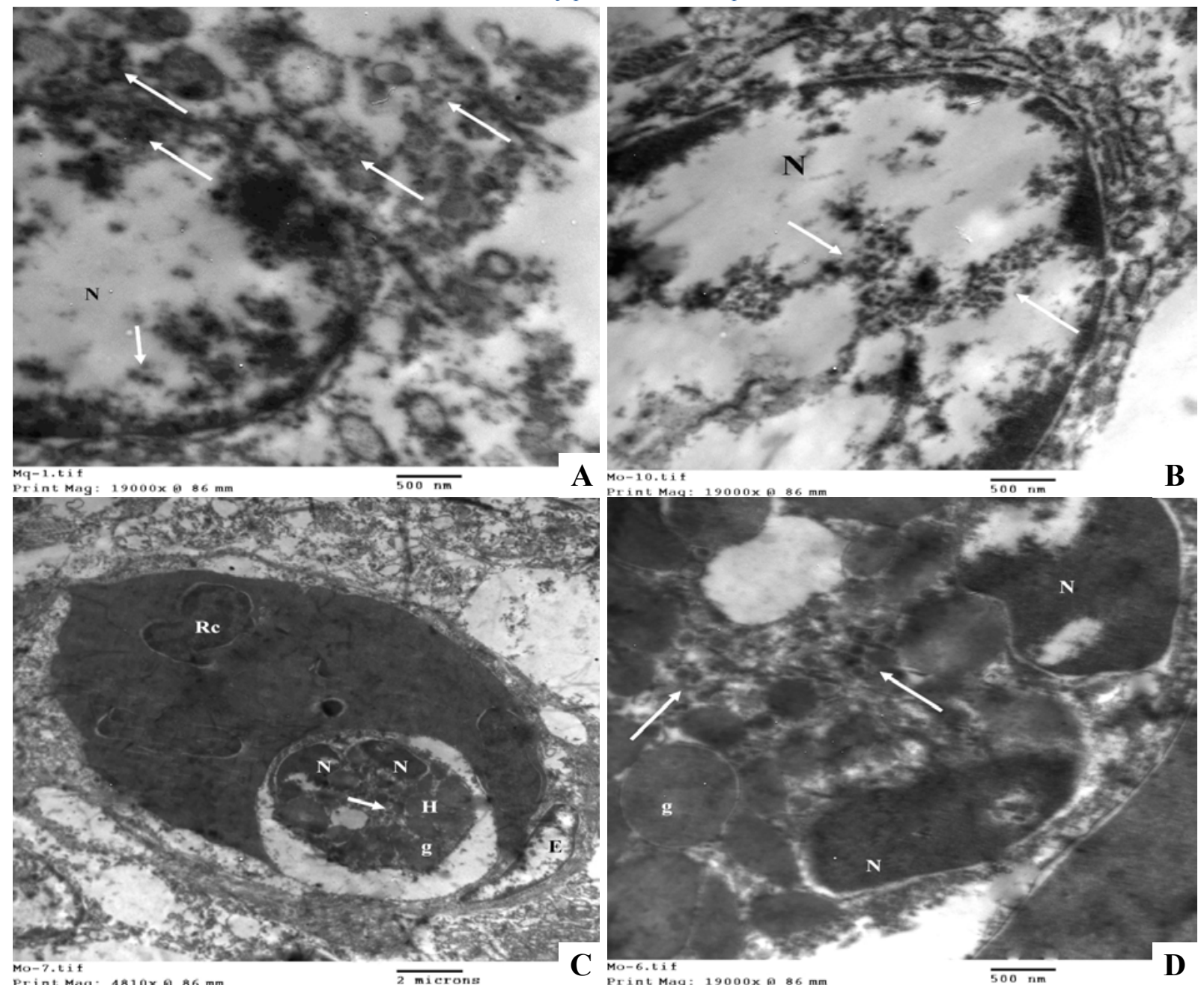

Figure 5. Transmission electron micrograph of the trachea. A. The tracheal epithelial mucosa shows the presence of electron-dense viral particles surrounded by a hollow zone in the nucleus $(\mathrm{N})$ and cytoplasm (white arrows). B. Tracheal mucosa showing presence of intranuclear (N) inclusion body inform of aggregation of electron dens viral particles surrounded by hallow zone (white arrow). C. Heterophil cell (H) in blood vessel of the tracheal mucosa showing a lobulated nucleus $(\mathrm{N})$, lysosomal granules $(\mathrm{g})$ and presence of electron-dense viral particles surrounded by a hollow zone in the cytoplasm (white arrow). D. A higher magnification of (C) demonstrating the viral particles.
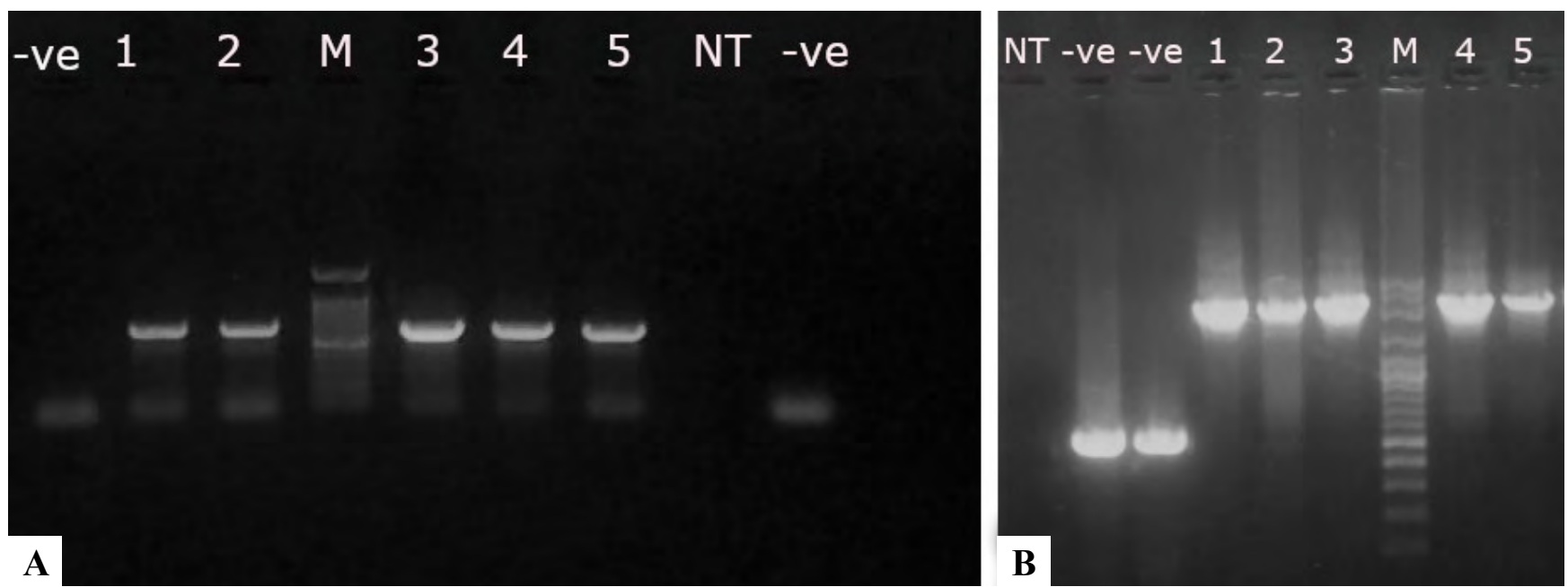

Figure 6. Gel electrophoresis image showing A. positive bands at 2.24-kbp for the TK gene and B. positive bands at 1.39-kbp portion for the gJ gene (NT: non-template, -ve: negative control, 1-5 clinical samples, M: DNA marker). 


\section{Discussion}

Outbreaks of ILTV infection can result in extensive economic losses reflected by high morbidity, mortality, and decreased egg production in laying hens (13).

In the present study, we performed a survey on ILTV infections in poultry farms in Egypt during the period from 2018 to 2020 and identified birds with severe respiratory signs with expectoration of blood. In these commercial chicken flocks in Egypt, the viral infection was associated with a high mortality rate. On postmortem examination, we found evidence of bloody cast in the tracheal lumen, which was suggestive of an outbreak of ILTV infection.

The clinically examined birds had conjunctivitis and respiratory signs manifestations including blood expectoration. The lumen of all segments of the trachea and larynx exhibited from mild mucoid exudate to bloody exudate, as previously reported by Gowthaman et al. (10) and Yavuz et al. (34). The noted intraluminal caseous and bloody casts could cause gasping and asphyxia of affected birds, which again could be a cause of death of the birds (25).

We consider the anatomopathological examination as the primary diagnostic tool and plays an important role in the diagnosis of ILTV infection, particularly in the acute phase of the viral infection by observation of multinucleated syncytial cells containing intranuclear eosinophilic inclusion bodies, a pathognomonic finding for ILTV infection $(23,34)$.

Histopathological examination of the larynx, trachea, lungs, and air sacs showed epithelial sloughing, deciliation, heterophilic infiltration, and the presence of syncytial cells with intranuclear inclusion bodies which were found in. These findings were exactly similar to Crespo et al. (6) study

Gross, histopathological examination and immunohistochemistry used in this study showed high affinity of ILTV to trachea and lung followed by air sac as mentioned in table 4,5 that can be explained by Kirkpatrick et al. (2006) and Yilmaz et al. (35) studies. They reported that field strains differed in their relative ability to induce mortality, clinical signs, and lesions in different organs. Some strains demonstrated high affinity for the trachea and little affinity for conjunctiva and, other strains showed high affinity for conjunctiva and lower affinity for trachea as well as low-virulent ILTV strain, which resulted in mild peribronchiolar edema, congestion of blood vessels with moderate infiltration of mononuclear cells.

In this study, the examined pulmonary and tracheal tissues showed congestion, hemorrhage, and perivascular edema, which findings are in line with those reported by Sivaseelan et al. (30) due to rupture of reticulin fibers, dissociation of cells, and disruption of blood vessels of the respiratory organs. Severe acute fibrinous bronchopneumonia revealed diffuse fibrinous exudate admixed with heterophils in the bronchial wall, extending to the interstitial tissue, parabronchus, and pleura. Moreover, syncytial cells were observed in the intraluminal exudate of the bronchi and parabronchus, extending to the pleura and air sacs as explained by Wang et al. (32) who also reported high ILTV loads in the throat, cecum, lung, and trachea as evidenced by qPCR data.

In the present study, the immunohistochemical analysis was showed a high sensitivity through the detection of immunoreactivity and the distribution of ILTV antigen in the syncytial cells, inflammatory cells and epithelial cells of larynx, trachea and lung is used to confirm the occurrence of the disease allowing visualization of viral antigen distributed in different tissues cannot be predicted by PCR as mentioned by Fuchs et al. (7). Highest IHC positivity has always been found in the trachea and lung as reported by Yavuz et al. (34).

The TEM can be used for rapid detection of high-titer ILTV infection (3.5 log 10) performed on tracheal scrapings. Diagnosis is based on visualization of herpesvirus particles in the studied tissue samples (15). Our results revealed the presence of viral particles in the tracheal mucosa, including the epithelial cells, and in heterophils inside the tracheal blood vessels as illustrated in Fig. 5 as well as presence of intranuclear inclusions bodies in tracheal lining epithelium.

Reports on ILT viremia are scarce (14); however, our study confirmed the presence of infected heterophils which may play a role in transferring the virus to non-respiratory tissues in affected birds but further studies need to be carried out.

Anatomopathological and histopathological findings revealed that examination of larynx, trachea, lung and air sac important in diagnosis of ILT. Forementioned in table 4,5 showed the alternation in these examined tissues. Also, IHC results allowed visualization of viral particles that was a useful complementary technique for diagnosis as in Egypt and developing countries it is cheaper and rapid than PCR.

Outbreaks of ILTV strains were detected in different countries such as in Egypt and Australia which may prompt the vaccine to revert to the virulent form and emergence of new recombinant ILTV strains $(4,28)$, while previous studies have documented severe ILT infections after administration of CEO vaccines (9) that may clarified the severe clinical signs of ILT were reported and confirmed with histopathology, IHC and molecular detection from sampled farms nevertheless their vaccination. However, it could be related to latent infection as Guy et al. (12) and Hughes et al. (16) documented that latent infection develops where the virus resides in the trigeminal ganglion one week after acute infection. This leads to the presence of carrier birds in the flock, which act as a continuous source of virus shedding and horizontal transmission of infection to other susceptible birds.

Overall, birds with severe and very severe lesions contributed to the highest proportion of samples $(67.48 \%)$. Highest lesions score of tracheas were detected in different farms of as Qalyubia then fayoum and sharkia governorates as shown in Fig. 4, which may be attributed to poor biosecurity measures were noted in the sampled farms showing with no 
adequate distance between them, high density of birds and poor ventilation as well as different vaccination protocols were used by the farms in this studied region.

The increased severity of ILTV lesions detected in different governates may be related to extensive and unguided administration of live attenuated CEO and TCO vaccines that occasionally revert to the virulent form following bird-to-bird passage in vaccinated flocks that need further molecular investigation and pathogenicity studies for the circulating ILTV strain in Egypt.

In conclusion, gross lesions besides the histopathological examination are key tools for preliminary ILT diagnosis. IHC and molecular detection confirm the specific diagnosis. Our findings showed variable degrees of ILTV severity in Egyptian farms. Detection of viral antigen with IHC technique was an advantage compared to PCR due to lower cost. Poor biosecurity and management gave rise to recrudescence of ILT infection that emphasizes the importance of good biosecurity level to be considered.

\section{Conflict of interest}

Authors declare there are no competing interests.

\section{References:}

1. Agnew-Crumpton R, Vaz PK, Devlin JM, O'Rourke D, Blacker-Smith HP, Konsak-Ilievski B, Noormohammadi AH. Spread of the newly emerging infectious laryngotracheitis viruses in Australia. Infection Genetics Evolution. 2016;43: 67-73.

2. Ali A, Mansour S, Mohamed M, Ali H, Shahin A. Molecular characterization of thymidine kinase and glycoprotein $\mathrm{G}$ genes from a possible vaccine induced infectious laryngotracheitis outbreak in Egypt. Pak. Vet. J.2014;34: 381-385

3. Bancroft JD, Suvarna SK, Layton C. Bancroft's Theory and Practice of Histological Techniques, Expert Consult: Online and Print, 7: Bancroft's Theory and Practice of Histological Techniques. Elsevier Health Sciences. 2013.

4. Bayoumi M, El-Saied M, Amer H, Bastami M, Sakr EE, El-Mahdy M. Molecular characterization and genetic diversity of the infectious laryngotracheitis virus strains circulating in Egypt during the outbreaks of 2018 and 2019.Arch Virol.2020;165(3):661-670.

5. Craig MI, Rojas MF, van der Ploeg CA, Olivera V, Vagnozzi AE, Perez AM, König GA. Molecular Characterization and Cluster Analysis of Field Isolates of Avian Infectious Laryngotracheitis Virus from Argentina. Front. Vet. Sci. 2017; 4(December), 1-7.

6. Crespo R, Woolcock PR, Chin RP, Shivaprasad HL, Garcia M. Comparison of diagnostics techniques in an outbreak of infectious laryngotracheitis from meat chickens. Avian Dis.2007; 51:858-862.
7. Fuchs W, Veits J, Helferich D. et al. Molecular biology of avian infectious laryngotracheitis virus. Vet Res.2007; 20:261-279

8. Garcia M, Riblet SM. Characterization of infectious laryngotracheitis virus isolates: demonstration of viral subpopulations within vaccine preparations.Avian Dis. 2001;45:558-66.

9. García M. Current and future vaccines and vaccination strategies against infectious laryngotracheitis (ILT) respiratory disease of poultry. Vet Microbiol. 1;206:15762. 2017.

10. Gowthaman V, Kumar S, Koul M, Dave U, Murthy Trgk, Munuswamy P, Joshi SK. Infectious laryngotracheitis: Etiology, epidemiology, pathobiology, and advances in diagnosis and control-a comprehensive review. Vet. Quart. 2020; 40(1), 140-161.

11. Guy JS, Bagust TJ. Laryngotracheitis. In: Diseases of Poultry, 11th ed. Y. M. Saif, H. J. Barnes, J. R. Glisson, A. M. Fadly, L. R. McDougald, and D. E. Swayne, eds. Iowa State University Press, Ames, IA. 2003. pp. 121-134.

12. Guy JS, Barnes HJ, Smith L. Increased virulence of modified-live infectious laryngotracheitis vaccine virus following bird-to- bird passage. Avian Dis.1991; 35:348-355.

13. Guy JS, Garcia M. Laryngotracheitis. In Y.M. Saif, A.M. Fadly, J.R. Glisson, L.R. McDougald, L.K. Nolan \& D.E. Swayne (Eds.), Diseases of Poultry 12th edn (pp. 137-152). Ames, IA: Blackwell Publishing Professional.2008.

14. Hidalgo H. Infectious Laryngotracheitis: A Review. Rev Bras Cienc Avic. 2003; 5: 157-68.

15. Hughes CS, Jones RC. Comparison of cultural methods for primary isolation of infectious laryngotracheitis virus from field materials. Avian Pathology. 1988; 17: 295-303

16. Hughes CS, Williams RA, Gaskell RM. et al. Latency and reactivation of infectious laryngotracheitis vaccine virus. Arch Virol.1991; 121:213-218

17. Kirkpatrick NC, Mahmoudian A, O’Rourke D, Noormohammadi AH. Differentiation of infectious laryngotracheitis virus isolates by restriction fragment length polymorphic analysis of polymerase chain reaction products amplified from multiple genes. Avian dis. 2006; 50(1), 28-33

18. Koski DM, Predgen AS, Trampel DW, Conrad SK, Narwold DR, Hermann JR. Comparison of the pathogenicity of the USDA challenge virus strain to a field strain of infectious laryngotracheitis virus. Biologicals.2015; 43(4), 232-237.

19. Lee SW, Markham PF, Coppo MJC, Legione AR, Markham JF, Noormohammadi AH, Browning GF, Ficorilli N, Hartley CA, Devlin JM. Attenuated vaccines recombine to form virulent field viruses. Science.2012; 337,188

20. Menendez KR, García M, Spatz S, Tablante NL. Molecular epidemiology of infectious laryngotracheitis: 
a review Avian Pathology.2014; 43, No. 2, 108-117

Braz J Vet Pathol, 2021, 14(2), 88 - 98

DOI: $10.24070 /$ bjvp.1983-0246.v14i2p88-98

21. Oldoni I, Rodriguez-Avila A, Riblet SM, Zavala G, Garcia M. Pathogenicity and growth characteristics of selected infectious laryngotracheitis virus strains from the United States. Avian Pathology.2009; 38, 47-53.

22. Ou SC, Giambrone JJ, Macklin KS. Comparison of a TaqMan real-time polymerase chain reaction assay with a loop-mediated isothermal amplification assay for detection of Gallid herpesvirus 1. J Vet Diagn Invest.2012; 24:138-141

23. Preis I, Fiúza A, Silva C. et al. Pathological, immunohistochemical, and molecular findings in commercial laying hens and in backyard chickens naturally infected with the infectious laryngotracheitis virus. Braz J Poult Sci.2014; 16:359-366

24. Preis IS, Braga JFV, Couto RM, Martins NR, Ecco R. Outbreak of infectious laryngotracheitis in large multiage egg layer chicken flocks in Minas Gerais, Brazil. Pesquisa Veterinária Brasileira.2013;33(5):591- 96.

25. Reddy VRAP, Steukers L, Li Y, Fuchs W, Vanderplasschen A, Nauwynck HJ. Replication characteristics of infectious laryngotracheitis virus in the respiratory and conjunctival mucosa. Avian Pathology. 2014; 43(5), 450-457.

26. Rodriguez-Avila A, Oldoni I, Riblet S, García M. Replication and transmission of live attenuated infectious laryngotracheitis virus. Avian Diseases.2007; 51, 905-911.

27. Roizman B. Herpesviridae. In: Virology, 3rd ed. Fields, B. N., D. M.Knipe, and P. M. Howley, eds. LippincottRaven, Philadelphia, PA. 1996.pp. 2221-2230.

28. Sabir AJ, Olaogun OM, O'Rourke D, Fakhri O, Coppo MJC, Devlin JM, Konsak-Ilievski B, Noormohammadi $\mathrm{AH}$. Full genomic characterisation of an emerging infectious laryngotracheitis virus class $7 \mathrm{~b}$ from Australia linked to a vaccine strain revealed its identity. Infect Genet E.2020; 78:104067.

29. Shehata AA, Halami MY, Sultan HH, Abd El-Razik AG, Vahlenkamp TW. Chicken embryo origin-like strains are responsible for infectious laryngotracheitis virus outbreaks in Egyptian cross-bred broiler chickens. Virus Genes. 2013; 46(3), 423-430.

30. Sivaseelan S, Rajan T, Malmarugan S, Balasubramaniam GA, Madheswaran R. Tissue tropism and pathobiology of infectious laryngotracheitis virus in natural cases of chickens. Israel Journal of Veterinary Medicine.2014; 69(4), 197-202.

31. Tantawy HH, El Batrawy AM, Bastami, M.A. et al. Avian infectious laryngo-tracheitis in Egypt. Epidemiology, virus isolation and infection. Vet Res Commun.1983; 6:281-287

32. Wang LG, Ma J, Xue CY, Wang W, Guo C, Chen F, Cao YC. Dynamic distribution and tissue tropism of infectious laryngotracheitis virus in experimentally infected chickens. Archives of Virology.2013; 158(3),
659-666. https://doi.org/10.1007/s00705-012-1414-8

33. Williams RA, Bennett M, Bradbury JM et al. Demonstration of sites of latency of infectious laryngotracheitis virus using the polymerase chain reaction. J Gen Virol.1992; 73:2415-2420

34. Yavuz O, Özdemir Ö, Aras Z, Terzi F. Immunohistochemical studies on infectious laryngotracheitis in the respiratory tract lesions in naturally infected laying hens. Kafkas Univ Vet Fak Derg.2018; 24:257-264. https://doi.org/10.9775/ kvfd.2017.18805

35. Yilmaz F, Timurkaan N, Bulut H. Detection of infectious laryngotracheitis virus in trigeminal ganglia by avidin-biotin complex method in chickens: Short communication. Acta Veterinaria Hungarica.2004; 52(2), 167-171. 